University of Wollongong

Research Online

Faculty of Engineering and Information

Faculty of Engineering and Information

Sciences - Papers: Part A

Sciences

$1-1-2012$

\title{
Evaluating disaster management knowledge model by using a frequency- based selection technique
}

Siti Hajar Othman

University of Wollongong, sho492@uow.edu.au

Ghassan Beydoun

University of Wollongong, beydoun@uow.edu.au

Follow this and additional works at: https://ro.uow.edu.au/eispapers

Part of the Engineering Commons, and the Science and Technology Studies Commons

Research Online is the open access institutional repository for the University of Wollongong. For further information contact the UOW Library: research-pubs@uow.edu.au 


\title{
Evaluating disaster management knowledge model by using a frequency-based selection technique
}

\begin{abstract}
Disaster Management (DM) is a multidisciplinary endeavour and a very difficult knowledge domain to model. It is a diffused area of knowledge that is continuously evolving and informally represented. Metamodel is the output artefact of metamodelling, a software engineering approach, which makes statements about what can be expressed in the valid models of the knowledge domain. It is an appropriate high level knowledge structure to facilitate it being communicated among DM stakeholders. A Disaster Management Metamodel (DMM) is developed. To satisfy the expressiveness and the correctness of a DMM, in this paper we present a metamodel evaluation technique by using a Frequencybased Selection. The objective of this technique is to evaluate the importance of the individual concepts used in the DMM, thus, the quality of the metamodel can be measured quantitatively.
\end{abstract}

\section{Keywords}

evaluating, model, disaster, frequency, selection, technique, management, knowledge

Disciplines

Engineering | Science and Technology Studies

\section{Publication Details}

Othman, S. Hajar. \& Beydoun, G. (2012). Evaluating disaster management knowledge model by using a frequency-based selection technique. Lecture Notes in Computer Science, 7457 21-27. 


\title{
Evaluating Disaster Management Knowledge Model by Using a Frequency-Based Selection technique
}

\author{
Siti Hajar Othman ${ }^{1,2}$ and Ghassan Beydoun ${ }^{2}$ \\ ${ }^{1}$ Faculty of Computer Science and Information System, \\ Universiti Teknologi Malaysia, 81310 Skudai, Johor, Malaysia \\ ${ }^{2}$ School of Information Systems and Technology, Faculty of Informatics, \\ University of Wollongong, Wollongong NSW 2522, Australia \\ \{sho492, beydoun\}@uow.edu.au
}

\begin{abstract}
Disaster Management (DM) is a multidisciplinary endeavour and a very difficult knowledge domain to model. It is a diffused area of knowledge that is continuously evolving and informally represented. Metamodel is the output artefact of metamodelling, a software engineering approach, which makes statements about what can be expressed in the valid models of the knowledge domain. It is an appropriate high level knowledge structure to facilitate it being communicated among DM stakeholders. A Disaster Management Metamodel (DMM) is developed. To satisfy the expressiveness and the correctness of a DMM, in this paper we present a metamodel evaluation technique by using a Frequency-based Selection. The objective of this technique is to evaluate the importance of the individual concepts used in the DMM, thus, the quality of the metamodel can be measured quantitatively.
\end{abstract}

Key words: Frequency-based Selection, Metamodel, Disaster Management, Knowledge Model, Model Transformation

\section{Introduction}

Knowledge is information presented within a particular context, yielding insight into actions taken in the context [5]. The effectiveness of a knowledge model depends on the abstraction effectiveness of individual concepts used to describe the domain [1]. The richer the meaning attached to the concepts, the less time a modeller requires to operationalise the model [2]. The meaning and definition of concept terminologies and their relationships are not only domain specific but may even differ from one observer to another [3, 4]. A challenge in creating a new model and identifying the domain concepts is resolving ambiguity and inconsistencies of domain terminologies. A model synthesis process adapts the software engineering practice, 'Metamodelling' and provides means to reconcile the inconsistencies across observers. This is a modular and layered process typically used to endow a well-established methodology or a modelling language with an abstract notation, discerning the abstract syntax and semantics of the modelling elements. By focussing on the evaluation and the metamodelling process on Disaster Management, this paper makes a significant contribution using metamodelling to unify key concepts into a metamodel that can be used as knowledge sharing platform. Later, this artefact can be reused by DM 
stakeholders to develop their DM customised models by retrieving parts and components of previous solutions to suit their current needs (disaster on hand). DM knowledge can be viewed from different lenses (e.g.: Know What, Know Who, Know How, Know Where, Know Why...) and understanding them is required to support its structuring. Structuring the Disaster Management (DM) knowledge requires understanding of its environment and elements (organisational, operations, processes or stakeholders). DM knowledge is also scattered in public resources such as the internet, books, online databases, libraries, newspapers or pamphlets. How this knowledge is applied in new situations is rarely explored [6]. Indeed, reusing and sharing knowledge is a form of knowledge creation and as pointedly stated in Beerli et. al [7 pp.3]: "Knowledge can be regarded as the only unique resource that grows when shared, transferred and skilfully managed." By developing an appropriate high level knowledge structure for this domain through a metamodel, a DM modelling knowledge is identified.

A metamodel identifies domain features and related concepts (as any other model) and is created with the intent to formally describe the semantics underpining a formal modelling language [8]. Without a metamodel, semantics of domain models can be ambiguous. In metamodel, concept and relationships are two important elements. A concept characterizes domain entities and relationships characterizes links between them [9]. Metamodel must form true or faithful representations so that queries of a model give reliable statements about reality, or manipulations of the model result in reliable adaptations of reality. A metamodel requires evaluation to satisfy the requirement of generality, expressiveness and completeness of the artefact. With respect to this, this paper presents how the Frequency-Based Selection (FBS) is used to evaluate the DM metamodel. The rest of this paper is structured as follows: Section II describes the related work on disaster management, metamodel evaluation in metamodelling and the DMM. Section III presents the actual evaluation of FBS against the DM metamodel. Section IV presents result of the evaluation and Section V concludes the paper with a discussion on our findings and future work.

\section{Related Works}

In this section, the related works on disaster management knowledge, metamodel evaluation in a metamodelling environment and a DM metamodel are discussed before the actual implementation of FBS technique is presented.

\subsection{Disaster Management Knowledge}

Disaster Management (DM) aims to reduce or avoid the potential losses from hazards, assure prompt and appropriate assistance to victims of disaster and achieve rapid and effective recovery. The United Nation (UN) recognises at least 40 types of disasters and classifies them into two types of disasters including: natural and technological/man-made disasters. Knowledge applied in this domain changes across various phases of a disaster. Standard DM phases include mitigation, preparedness, response and recovery [10]. Structuring the DM knowledge requires understanding of its environment and elements (organisational, operations, processes or stakeholders). There are varieties of DM models which have been developed by many stakeholders 
(researchers, government or non-government agencies, community and individuals). These models can broadly be grouped according to seven main perspectives: disaster phase oriented (e.g.: recovery or preparedness stage), organisation oriented (e.g.: Red-Cross coordination, State Police arrangement during emergency, User/Role oriented (e.g.: volunteers, hospitals, aid agencies), disaster type oriented (e.g.: earthquake, disease infection), technology oriented (e.g.: GIS, Satellite for disaster monitoring), disaster activity oriented (e.g.: evacuation, search and rescue) or decision type oriented (e.g.: reasoning technique for disaster decision making). In developing a metamodel specific to this challenging domain, typically, the first question that will be asked after any metamodel is successfully developed is how the metamodel is relevant to its real application domain. Therefore, evaluation to the artefact is crucial.

\subsection{Metamodel Evaluation and its transformation in a Metamodelling Approach}

The quality of the metamodel is measured based on how the metamodel can fulfil the purpose of its development [11]. In other words, the created metamodel has to respond to the needs of the domain practitioners. This includes increasing the transparency to the knowledge encoded within the domain applications and be able to be validated by relevant experts in the domain. three motivations of why metamodel requires evaluation are: (i) initial domain literatures used to develop the metamodel is sometimes not complete, therefore it is necessary to fill in some blanks with hypothesis unsupported by the initial literature; (ii) domain literature is not always coherent, hence when creating a metamodel it might be inescapable to make controversial choices; (iii) metamodeler might be biased, thus when creating a metamodel, he or she might unwillingly create distortions [12].

In metamodelling, metamodels and models relate through model transformation [13]. During metamodel evaluation, model transformations are explored and evaluated. Model transformation is one of a process of converting one model to another model in a metamodelling framework. Also, the acceptance of a system of metamodels for practical use depends on the validity of the metamodels and the transformations on a given abstraction hierarchy [14 pp. 163]. Model-to-model transformation is a key technology for Object Management Group (OMG)'s Model Driven Architecture [15] and underpins realising the various functionalities of DMM. DM solutions need to be transformed to DMM during knowledge storage and DMM needs to be transformed back to various DM solution models by DM users later. This research follows the modelling abstraction offered by Meta Object Facility (MOF) framework in performing a transformation of metamodel-to-model for DMM. The MOF framework provides a capability to support different types of metadata in its four meta-layers: User Model level (M0), Model level (M1), Metamodel level (M2), and Meta-metamodel (M3) and can be used to define different information models. Model transformation in MOF (presented in Figure 1) can be viewed in vertical and horizontal dimensions [16].

A horizontal transformation involves transforming a model into a target model at the same level of modelling abstraction. This is true no matter how high or low the artefact modelling abstraction level is [16]. Semantics of horizontal transformations is applied in this paper when DMM is horizontally transformed to produce its new 
updated version after performing the FBS technique against the metamodel. A Vertical transformation presents the transformation of model from one level to a different level of modelling abstraction. The transformation can either be from an upper to a lower level (e.g.: from metamodel (M2) level to model (M1) level), or conversely from a lower to an upper level (e.g.: from model level (M1) to metamodel level (M2)). The vertical transformation is performed when "the DM model and DM User Model are being derived from its conformant DMM (metamodel)". The process of deriving individual concepts in the models is also vertical transformations.

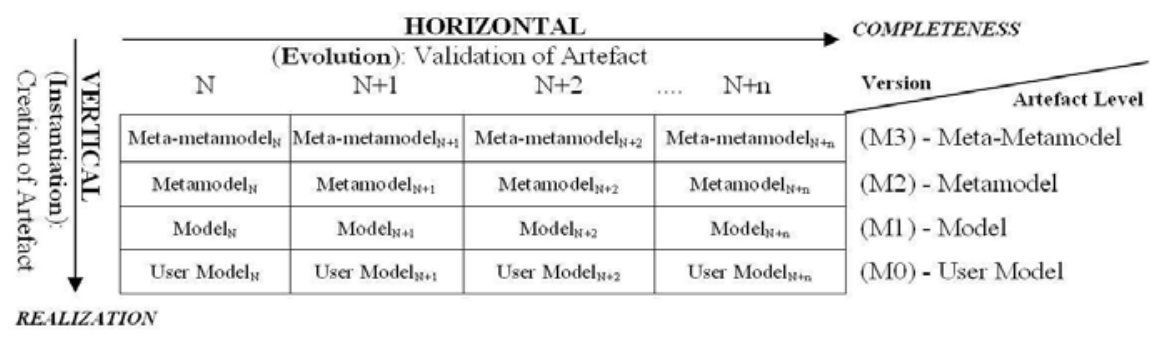

Fig. 1. Horizontal and vertical model transformation in MOF metamodelling

\subsection{The Disaster Management Metamodel}

The DMM is the output of the metamodelling approach applied in this paper. It will serve as a representational layer to enable appropriate domain modelling and knowledge storage relating to different DM activities and disaster scenarios. It is a DM specific language developed by using the 8 step Metamodelling Creation Process adapted from Beydoun et al. [17, 18]. In [19], this initial DMM is developed and uses DMM1.0 as its version. The metamodel is presented in four sets of concept classes: the Mitigation, Preparedness, Response and Recovery class of concepts. Each set represent a corresponding DM phase and clearly describes the DM domain to its users. This initial metamodel has been first evaluated in [20] by using a 'Comparison against other models' technique. The aim of the first evaluation is to identify any missing concepts in the metamodel and to also ensure its broad coverage. Result from the first evaluation changes the DMM1.0 to its updated version, a DMM1.1. Normally a metamodel requires iterative evaluation in its development because it needs to achieve different quality goal in each evaluation cycle. In this paper, with the aim to evaluate the importance of the individual concepts included in DMM, this time the DMM is validated for a second cycle by using the FBS technique. Result derived from the evaluation conducted in this paper creates the DMM1.2 version.

To visibly show the changes occurred before and after performing the FBS, this paper uses the Mitigation-phase and the Response-phase class of concepts as the metamodel samples, presented in the Figure 2 and Figure 3 respectively. In both classes, concepts and their relationships are depicted. The following shows the list of concepts used in each DMM classes:

i) DMM Mitigation concepts: MitigationPlan, MitigationOrganisation, MitigationTask, NeedsPlanning, BuildingCodes, Land-UsePlanning, InformationUpdates, MitigationGoal, RiskReduction, People, Property, Lifeline, NaturalSite, HazardAssessment, RiskAnalysis, 
StructuralMitigation, Non-StructuralMitigation, Vulnerability, DisasterRisk, StrategicPlanningCommittee, Legislation, Insurance and Exposure;

ii) DMM Preparedness concepts: PreparednessPlan, PreparednessOrganisation, PreparednessTask, SuppliesRegistry, Warning, PreparednessGoal, Evacuation, BeforeDisaster, Event, DecisionMaking, Administration, EmergencyPublicInformation, Pre-Position, DisasterFactor, Exposure, DisasterRisk, Training, PreparednessTeam, Media, MutualAidAgreement, PublicEducation, PublicAwareness, Resource, Monitoring, AidAgency;

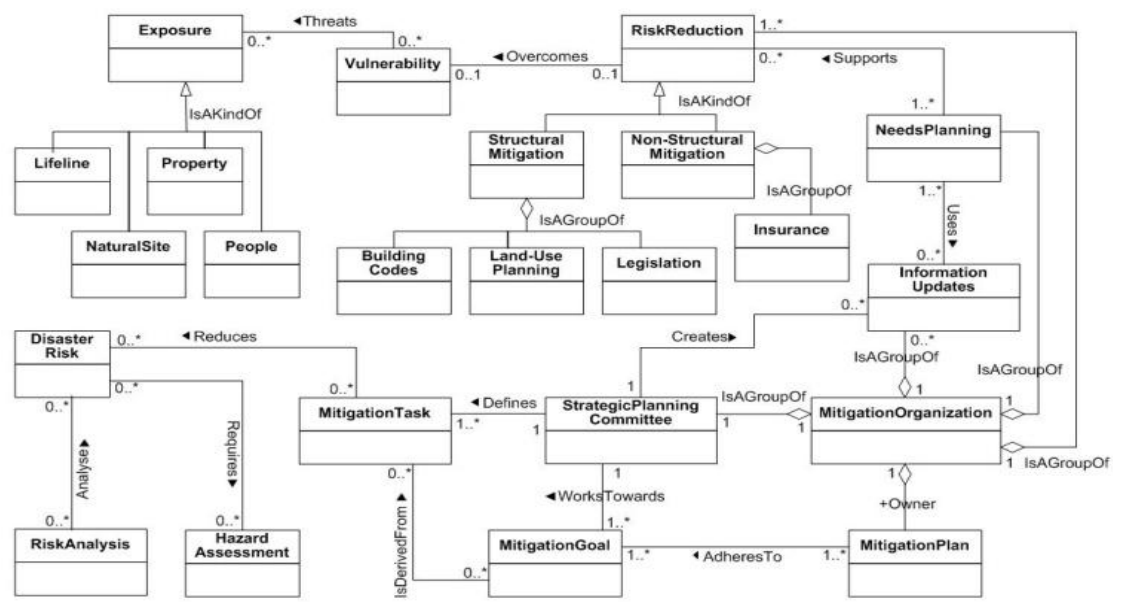

Fig. 2. The DMM1.1: The first validated version of Mitigation-phase class of concepts [20]

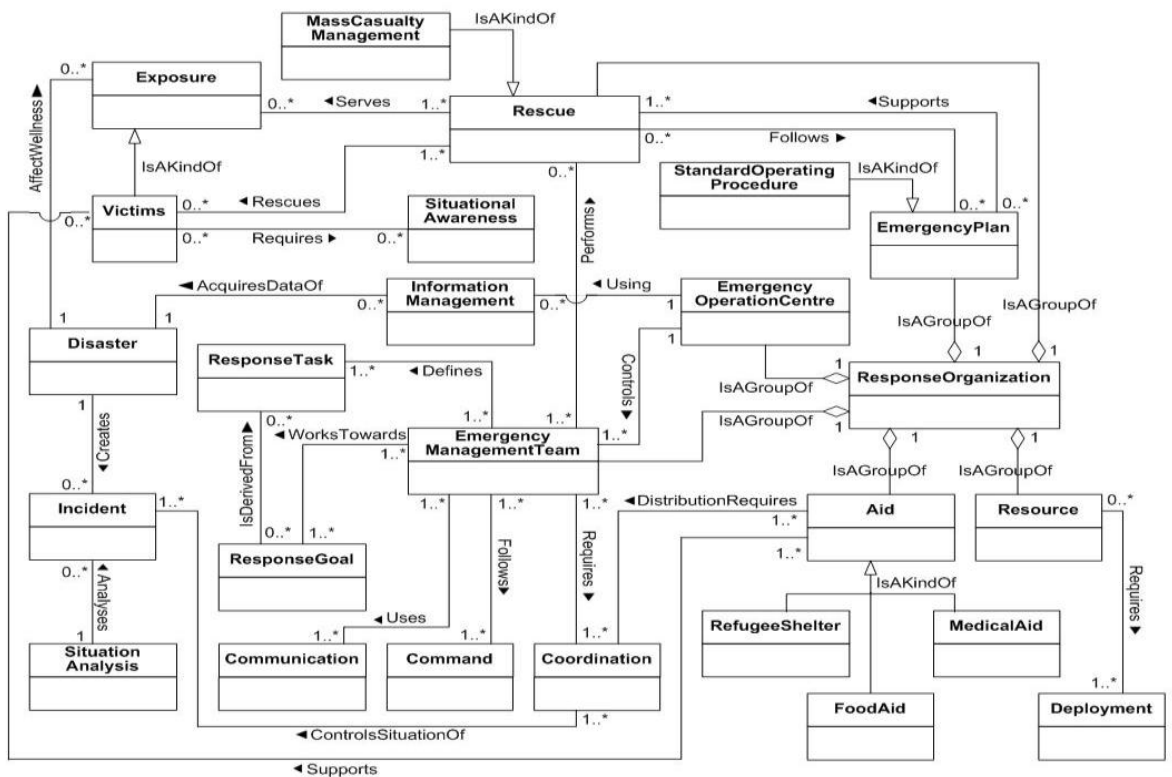

Fig. 3. The DMM1.1: The first validated version of Response-phase class of concepts [20] 
iii) DMM Recovery concept: RecoveryPlan, RecoveryOrganisation, RecoveryTask, Demobilization, LongTermPlanning, RecoveryGoal, Reconstruction, AfterDisaster, DamageAssessment, TaskReview, Resilience, Victim, EmergencyManagementTeam, Resource, DebrisRemoval, Effect, EconomicRestoration, Exposure, FinancialAssistence, MentalHealthRecovery, AidDistribution.

\section{Frequency-Based Selection Implementation}

In this section, FBS and a special frequency parameter used to estimate the importance of the individual concepts in the DMM, a Degree of Confidence (DoC) are described. This is then is followed by the representation of FBS actual implementation against the DMM. Result of this evaluation is presented in the next section.

\subsection{Frequency-Based Selection}

Frequency-Based Selection is a Feature Selection technique that evaluates the importance of individual concepts in the model developed [21]. It is based on the premise that the best model is formed using the most common features [22] and it is commonly used, for example, in data mining, software analysis, and medical retrieval systems. By performing FBS, features (concepts) that do not have correlations (or a need) to the classification are removed from DMM. The way FBS is adapted to validate the significance of DMM concepts acknowledges the five metamodel quality criteria described in [23]: a reasonable depiction (e.g. a statistical measure) of the relative 'importance' of candidate concepts; a predictive ability of the metamodel that is reasonably consistent with baseline models across the domain; the metamodel has independent meaningful variables; the metamodel highlights all input variables essential to describe critical components of a domain and the metamodel can provide a storyline to its users to tell how and why a derived model behaves as it does.

To perform FBS, 10 set of existing DM models of Set V2 is used (Table 1). The set is formed based on phase-specific and other perspectives (e.g.: role/user, operation, organisation, decision or technology-based DM models. For a selection, a model coverage values ( $\mathbf{R}_{\text {coverage }}$ ) are used: 0.3 is assigned to a model that has full coverage to all phases in DM (Mitigation, Preparedness, Response and Recovery phase). DM models with coverage less than 0.3 focuses on specific DM phases, activities or roles, as follows: 0.2 is assigned to coverage of models that can cover 2-3 DM phases in their models. 0.1 is set to a model that covers only one DM phase (any one of four DM phases) or a specific DM perspectives (e.g.: evacuation operation (operationbased), the roles of the disaster analyzer in disaster monitoring (user/role-based)). If a model does not cover any single DM phase fully, 0.0 is set to the model and will be excluded from any further investigation. This selection process ensures that all DMM concepts are tested against some concepts in the models selected. That is, each DMM concept is examined in a vertical model transformation. Where required, DMM is modified to ensure that it can represent all models in the validation sets (through a horizontal transformation). 
Table 1. A set of 10 DM models (Set V2) for an evaluation of DMM

\begin{tabular}{|c|l|c|c|l|}
\hline \multicolumn{1}{|c|}{ SET V2 } & Y $_{\text {published }}$ & $\mathbf{R}_{\text {coverage }}$ & \multicolumn{1}{|c|}{$\begin{array}{c}\text { Model coverage: } \\
\text { (Perspective) }\end{array}$} \\
\hline 1 & $\begin{array}{l}\text { Disaster Risk Management \& Mitigation Management, } \\
\text { [24] }\end{array}$ & 2006 & 0.3 & All Phases: (Activity-based) \\
\hline 2 & $\begin{array}{l}\text { Policies for Guiding Planning for Post-Disaster Recovery } \\
\text { and Reconstruction, [25] }\end{array}$ & 2005 & 0.2 & $\begin{array}{l}\text { Mitigation and Recovery: } \\
\text { (Management-based) }\end{array}$ \\
\hline 3 & Disaster Risk Management Working Concept, [26] & 2002 & 0.3 & All Phases: (Activity-based) \\
\hline 4 & $\begin{array}{l}\text { Disaster Information, Innovative Disaster Information } \\
\text { Service, [27] }\end{array}$ & 2008 & 0.3 & $\begin{array}{l}\text { All Phases: (Technological- } \\
\text { based) }\end{array}$ \\
\hline 5 & $\begin{array}{l}\text { Situation-Aware Multi-Agent System for Disaster Relief } \\
\text { Operations Management, [28] }\end{array}$ & 2006 & 0.2 & $\begin{array}{l}\text { Preparedness and Response: } \\
\text { (Technological-based) }\end{array}$ \\
\hline 6 & $\begin{array}{l}\text { An Approach to the Development of Commonsense } \\
\text { Knowledge for Disaster Management, [29] }\end{array}$ & 2007 & 0.3 & $\begin{array}{l}\text { All Phases: (Disaster and } \\
\text { Activity-based) }\end{array}$ \\
\hline 7 & Earthquake Protection, [30]. & 1992 & 0.3 & $\begin{array}{l}\text { All Phases: (Disaster and } \\
\text { Organisation-based) }\end{array}$ \\
\hline 8 & Disaster Stage and Management Model, [31] & 2008 & 0.3 & $\begin{array}{l}\text { All Phases: (Disaster-based } \\
\text { and Management-based) }\end{array}$ \\
\hline 9 & $\begin{array}{l}\text { Teaching Disaster Nursing by Utilizing the Jennings } \\
\text { Disaster Nursing Management Model, [32]. }\end{array}$ & 2004 & 0.3 & $\begin{array}{l}\text { All Phases: (User/Role- } \\
\text { based) }\end{array}$ \\
\hline 10 & Disaster Management - a Theoretical Approach, [33] & 2008 & 0.3 & All Phases: (Disaster-based) \\
\hline
\end{tabular}

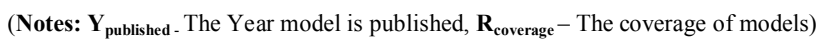

\subsection{The Degree of Confidence (DoC)}

Using the concept frequency, an importance value for each concept in DMM is estimated and expressed as the 'Degree of Confidence (DoC)'. This value designates the expected probability that a DMM concept is used in a randomly chosen disaster model. DoC is derived by dividing 'the frequency of how many times a concept appears in all the investigated models (Set V2)' with 'the total number of Set V2 models'. For this purpose, DoC is based on the list of concepts that appeared in the DMM1.1 (our metamodel after its first evaluation) and is defined as follows:

Degree of Confidence $($ DoC $)=\frac{\text { Frequency of Concept }}{\text { Total Model of Set V2 }} \times 100 \%$

\subsection{The FBS Evaluation against the DMM}

To perform the FBS technique on DMM, concepts to be verified from models in the evaluation Set V2 are first collated. This is to ensure that these concepts can all be refined using DMM1.1. As described in Section 3.1, Set V2 is a selection of DM models that have a wider DM coverage. Specialised DM models will naturally focus on a specific DM phase and naturally omit the use of some concepts. Therefore using models with wider coverage will provide a better indication on the frequency of concepts across the models. Their use will enable a frequency count of the individual DMM concepts. Concepts used in the models of Set V2 that are found similar and that are a refinement of DMM concepts are scored in this evaluation. The higher their score, the more important the concepts are deemed to the DM domain. Concepts that have a low score are revisited and are liable for deletion.

In applying FBS using the models in Set V2, DMM concepts that derive concepts of those models are identified. The frequency of usage of DMM concepts in those derivations is compiled and shown in Table 4 (for the Mitigation-phase concepts), Table 5 (for the Response-phase concepts). In what follows in this section, refinement 
of every model of Set V2 is overviewed. The outcome of FBS evaluation, leading to DMM1.2, is then presented in Section IV. Two models of Set V2 (Model 7 - The Organisation Model in Earthquake Disaster [30] and Model 9 - The Jennings Disaster Nursing Management Model [32] are used as the evaluation implementation samples of FBS.

\subsubsection{Sample FBS 1: Against the Organisation Model in Earthquake Disaster (Model 7 of Set V2)}

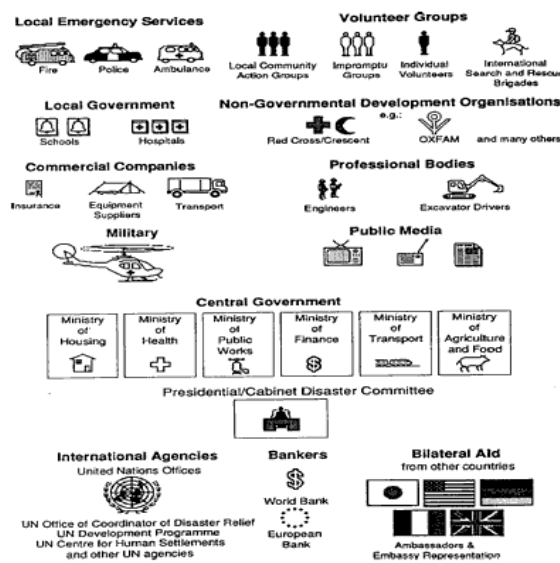

Fig. 4. The Organisation in Earthquake Disaster Model [30]

Reconstruction following an earthquake requires a renovation of the economy, jobs and income, daily life and social relations. Coburn [30] proposed that reconstruction tasks following an earthquake get organised sectorally (Figure 4). Coburn provides a few examples of how earthquake damage can be classified by sector and responsible organisations. Sectoral approach is advocated as different authorities have different responsibilities and reconstruction needs. As an example, for damages to schools, universities, and kindergartens including the number of lost classroom places and the loss of school equipment, become the responsibility of the Department of Education, Regional Education Authority, Private Education Institutions and the Department of Public Works of the country. As another example, any damage that may occur to agricultural building stock, loss of livestock, damage to equipment, vehicles, market gardening, greenhouses, food processing plants, food and produce storage becomes the responsibility of the Department of Agriculture and Food, Farming Organisations, Private Owners and Consumer Organisations. This model can be generated from the concepts RecoveryTask, RecoveryOrganisation and RecoveryGoal in DMM.

Evaluation against this model brings us to identify different ways to classify the Aid concept of DMM. A Bilateral Aid concept is found to not be covered in DMM. Thus, the Aid concept of DMM is split into three concepts: HumanitarianAid, DevelopmentAid and BilateralAid. The previous concepts that are used in previous DMM (FoodAid, MedicalAid and RefugeeShelter) were earlier grouped under HumanitarianAid. These changes are in the Response-phase of DMM. Following this, Table 2 shows the full list of Coburn model's concepts as derived from concepts in DMM. 
Table 2. Derive concepts in Coburn model by concepts in DMM

\begin{tabular}{|c|c|}
\hline DMM & Coburn model \\
\hline EmergencyManagementTeam & $\begin{array}{l}\text { - Local emergency services: Fire, Police, Ambulance } \\
\text { - International search and rescue brigade } \\
\text { - Professional Bodies: Engineers, Excavator drivers, Military }\end{array}$ \\
\hline Property & Local Government: School, Hospital \\
\hline People & Volunteer Groups: Local community action groups, Impromptu groups \\
\hline PreparednessTeam & Individual Volunteers \\
\hline AidAgency & $\begin{array}{l}\text { - Non-governmental development organisations: Red Cross/crescent } \\
\text { - International Agencies: United Nations Office, Bankers: World Bank }\end{array}$ \\
\hline Media & Public media \\
\hline Insurance & Insurance \\
\hline Resource & Commercial companies, Equipment Suppliers, Transport \\
\hline Aid & Bilateral Aid from other countries: Ambassadors, Embassy representation \\
\hline $\begin{array}{l}\text { RecoveryOrganisation, } \\
\text { RecoveryTask, RecoveryGoal }\end{array}$ & $\begin{array}{l}\text { Department of Agriculture and Food, Farming Organisations, Private Owners and } \\
\text { Consumers Organisations }\end{array}$ \\
\hline
\end{tabular}

\subsubsection{Sample FBS 2: Against the Jennings Disaster Nursing Management} Model (Model 9 of Set V2)

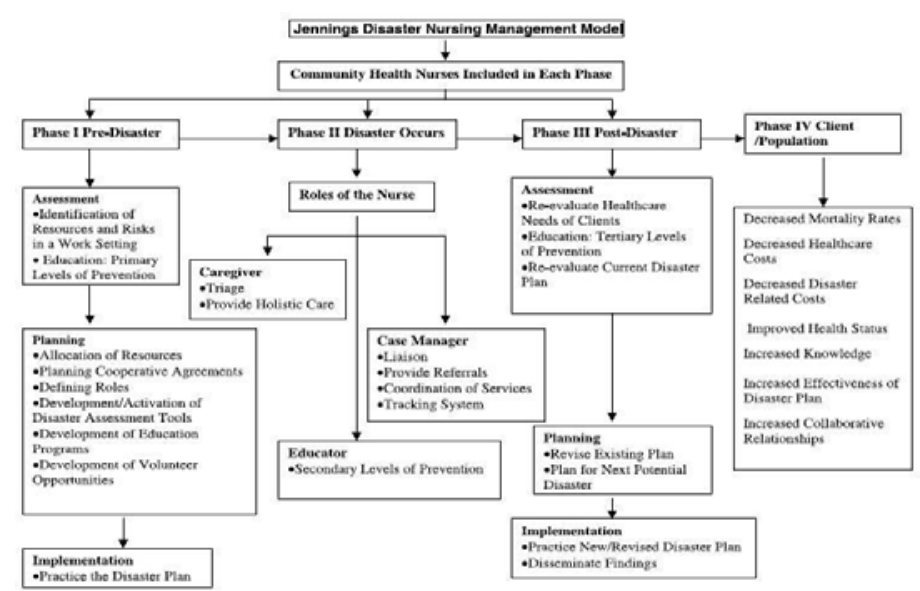

Fig. 5. Jennings Disaster Nursing Management Model [32]

The Jennings Disaster Nursing Management model [32] presented in Figure 5 defines nursing during DM as "the systematic and flexible ulitisation of knowledge and skills specific to disaster-related nursing, and the promotion of a wide range of activities to minimise the health hazards and life threatening damage caused by disasters in collaboration with other specialised fields". The model aims to help community nurses plan for and manage disasters in hospitals. There are four phases incorporated in the model: Phase 1 (Pre-Disaster), Phase II (Disaster), Phase III (Post-Disaster), and Phase IV (Positive Client/Population Outcomes). This model is taken to validate DMM concepts with the activities presented by the Jennings model. DMM can successfully derive all concepts in the Jennings model. The pre-disaster stage which is the first phase Jennings used in her model is identified clearly and represents the 
mitigation and preparedness-phase of the DMM. However the Jennings model disaster phase represents DMM's Response-phase and her post disaster represents DMM's Recovery-phase. The DMM concepts used to generate the Jennings model are shown in Table 3.

Table 3. Jennings Concepts Support for DMM Concepts

\begin{tabular}{|c|c|c|c|}
\hline \multicolumn{2}{|r|}{ JENNINGS Concepts } & \multicolumn{2}{|r|}{ DMM Concepts } \\
\hline Phase & Activity & Phase & Concept \\
\hline \multirow[t]{9}{*}{ Pre } & Identification of Resources and Risks & Mitigation & RiskAnalysis, HazardAssessment, \\
\hline & Education: Primary Level of Prevention & Preparedness & PublicEducation \\
\hline & Allocation of Resource & $\begin{array}{l}\text { Mitigation } \\
\text { Preparedness } \\
\text { Response } \\
\text { Recovery } \\
\end{array}$ & $\begin{array}{l}\text { NeedsPlanning } \\
\text { Pre-Position, SuppliesRegistry, } \\
\text { Deployment, } \\
\text { Demobilization }\end{array}$ \\
\hline & Planning Cooperative Agreement & Mitigation & StrategicPlanningCommittee \\
\hline & Defining Roles & Mitigation & StrategicPlanningCommittee \\
\hline & $\begin{array}{l}\text { Development/Activation of Disaster Assessment } \\
\text { Tools }\end{array}$ & Preparedness & Monitoring \\
\hline & Development of Education Programs & Preparedness & Public Education \\
\hline & Development of Volunteer Opportunities & Preparedness & PreparednessPlan \\
\hline & Practice the Disaster plan & Preparedness & Training \\
\hline \multirow[t]{7}{*}{ Occur } & Triage & Preparedness & Warning \\
\hline & Provide Holistic Care & Response & ResponseTask \\
\hline & Liaison & Response & EmergencyOperationCentre \\
\hline & Provide Referrals & Response & StandardOperatingProcedure \\
\hline & Coordination of Services & Response & Coordination, Command \\
\hline & Tracking System & Response & EmergencyOperationCentre \\
\hline & Secondary Level of Prevention & Response & Rescue \\
\hline \multirow[t]{6}{*}{ Post } & Re-evaluate Health Care & Recovery & TaskReview \\
\hline & Education: Tertiary Level of Prevention & Recovery & TaskReview \\
\hline & Re-evaluate Current Disaster Plan & Recovery & LongTermPlanning \\
\hline & Revise Existing Plan & Recovery & TaskReview \\
\hline & Plan for Next Potential Disaster & Recovery & LongTermPlanning \\
\hline & Disseminate Findings & Recovery & TaskReview \\
\hline
\end{tabular}

(Notes: MIT=Mitigation, $\mathbf{P R E}=$ Preparedness, $\mathbf{R E S}=$ Response, $\mathbf{R E C}=$ Recovery)

\section{The FBS Evaluation Result}

Table 4 and Table 5 respectively show the DoC values for all DMM concepts evaluated in the Mitigation and Response-phase class. The following five categories of concepts based on their DoC are defined: Very Strong (DoC result: $100-70 \%$ ), Strong (69-50\%), Moderate (49 - 30\%), Mild (29-11\%), and Very Mild (10 - 0 $\%$ ). Very Strong DoC is assigned to concepts that appear frequently in Set V2 models, whereas Very Mild is at the other end of the scale. For example, the DMM concept, MitigationPlan, has a DoC value of $90 \%$. It is expected that $90 \%$ of DM models with a mitigation phase will include it. It is also expected that $10 \%$ of DM models with a mitigation phase will not include it. For example, few models suggest forming a Strategic Planning Committee instead. Metamodel development is not about achieving perfection [34 pp. 23]. Aiming for a complete metamodel can lower its generalisability and has been cited as a common bad practice in metamodel development [34]. These views suggest that if a DMM concept is rarely used or needed, it may be better to delete it in some cases. As a result of this evaluation, 
concepts with zero DoC values are revisited and liable for deletion. For example, another DMM concept, BuildingCode, has a DoC value of 0 and is later revisited.

Table 4. Frequency result of Mitigation-phase concepts

\begin{tabular}{|c|c|c|c|c|c|c|c|c|c|c|c|c|}
\hline \multirow{2}{*}{\multicolumn{2}{|c|}{$\begin{array}{c}\text { DMM1.1 } \\
\text { Mitigation Concepts }\end{array}$}} & \multicolumn{10}{|c|}{ Model Set V2 } & \multirow{2}{*}{$\begin{array}{c}\text { Concept } \\
\text { Frequency }\end{array}$} \\
\hline & & 1 & 2 & 3 & 4 & 5 & 6 & 7 & 8 & 9 & 10 & \\
\hline 1 & MitigationPlan & $\sqrt{ }$ & $\sqrt{ }$ & $\sqrt{ }$ & $\sqrt{ }$ & $\sqrt{ }$ & $\sqrt{ }$ & & $\sqrt{ }$ & $\sqrt{ }$ & $\sqrt{ }$ & 9 \\
\hline 2 & MitigationOrganisation & $\sqrt{ }$ & $\sqrt{ }$ & $\sqrt{ }$ & $\sqrt{ }$ & $\sqrt{ }$ & $\sqrt{ }$ & $\sqrt{ }$ & $\sqrt{ }$ & $\sqrt{ }$ & $\sqrt{ }$ & 10 \\
\hline 3 & MitigationTask & $\sqrt{ }$ & $\sqrt{ }$ & $\sqrt{ }$ & $\sqrt{ }$ & $\sqrt{ }$ & & & & $\sqrt{ }$ & $\sqrt{ }$ & 7 \\
\hline 4 & NeedsPlanning & $\sqrt{ }$ & & $\sqrt{ }$ & & $\sqrt{ }$ & & & & $\sqrt{ }$ & & 4 \\
\hline 5 & InformationUpdates & & & & & $\sqrt{ }$ & $\sqrt{ }$ & & $\sqrt{ }$ & $\sqrt{ }$ & & 4 \\
\hline 6 & MitigationGoal & & & $\sqrt{ }$ & & $\sqrt{ }$ & $\sqrt{ }$ & & & $\sqrt{ }$ & $\sqrt{ }$ & 5 \\
\hline 7 & RiskReduction & $\sqrt{ }$ & $\sqrt{ }$ & $\sqrt{ }$ & $\sqrt{ }$ & $\sqrt{ }$ & $\sqrt{ }$ & & $\sqrt{ }$ & $\sqrt{ }$ & $\sqrt{ }$ & 9 \\
\hline 8 & People & & & & & $\sqrt{ }$ & & $\sqrt{ }$ & $\sqrt{ }$ & $\sqrt{ }$ & & 4 \\
\hline 9 & Property & & & & & & & & & & & $\mathbf{0}$ \\
\hline 10 & Lifeline & & $\sqrt{ }$ & & $\sqrt{ }$ & $\sqrt{ }$ & & & & & & 3 \\
\hline 11 & NaturalSite & & & & $\sqrt{ }$ & & & & & & & 1 \\
\hline 12 & HazardAssessment & & $\sqrt{ }$ & $\sqrt{ }$ & $\sqrt{ }$ & & $\sqrt{ }$ & $\sqrt{ }$ & $\sqrt{ }$ & $\sqrt{ }$ & $\sqrt{ }$ & 8 \\
\hline 13 & RiskAnalysis & $\sqrt{ }$ & $\sqrt{ }$ & $\sqrt{ }$ & $\sqrt{ }$ & $\sqrt{ }$ & $\sqrt{ }$ & & $\sqrt{ }$ & $\sqrt{ }$ & $\sqrt{ }$ & 9 \\
\hline 14 & StructuralMitigation & & $\sqrt{ }$ & & & & & & & & $\sqrt{ }$ & 2 \\
\hline 15 & Non-Structural Mitigation & & $\sqrt{ }$ & & & & & & & & $\sqrt{ }$ & 2 \\
\hline 16 & Vulnerability & & $\sqrt{ }$ & & & $\sqrt{ }$ & & & & $\sqrt{ }$ & $\sqrt{ }$ & 4 \\
\hline 17 & DisasterRisk & $\sqrt{ }$ & $\sqrt{ }$ & $\sqrt{ }$ & & & $\sqrt{ }$ & & $\sqrt{ }$ & $\sqrt{ }$ & & 6 \\
\hline 18 & StrategicPlanningOrganisation & $\sqrt{ }$ & & $\sqrt{ }$ & $\sqrt{ }$ & & $\sqrt{ }$ & & $\sqrt{ }$ & & $\sqrt{ }$ & 6 \\
\hline 19 & BuildingCodes & & & & & & & & & & & $\mathbf{0}$ \\
\hline 20 & Legislation & & $\sqrt{ }$ & & & $\sqrt{ }$ & & & & & & 2 \\
\hline 21 & Land-UsePlanning & & & & & & & & & & & $\mathbf{0}$ \\
\hline 22 & Insurance & & & & & & & $\sqrt{ }$ & & & & 1 \\
\hline
\end{tabular}

Table 5. Frequency result of Response-phase concepts

\begin{tabular}{|c|c|c|c|c|c|c|c|c|c|c|c|c|}
\hline \multirow{2}{*}{\multicolumn{2}{|c|}{\begin{tabular}{|c|} 
DMM1.1 \\
Response Concepts
\end{tabular}}} & \multicolumn{10}{|c|}{ Model Set V2 } & \multirow{2}{*}{$\begin{array}{l}\text { Concept } \\
\text { Frequency }\end{array}$} \\
\hline & & 1 & 2 & 3 & 4 & 5 & 6 & 7 & 8 & 9 & 10 & \\
\hline 1 & EmergencyPlan & $\sqrt{ }$ & $\sqrt{ }$ & $\sqrt{ }$ & $\sqrt{ }$ & $\sqrt{ }$ & $\sqrt{ }$ & $\sqrt{ }$ & $\sqrt{ }$ & $\sqrt{ }$ & $\sqrt{ }$ & 10 \\
\hline 2 & ResponseOrganisation & $\sqrt{ }$ & $\sqrt{ }$ & $\sqrt{ }$ & $\sqrt{ }$ & $\sqrt{ }$ & $\sqrt{ }$ & $\sqrt{ }$ & $\sqrt{ }$ & $\sqrt{ }$ & $\sqrt{ }$ & 10 \\
\hline 3 & ResponseTask & $\sqrt{ }$ & $\sqrt{ }$ & $\sqrt{1}$ & & $\sqrt{ }$ & & & & $\sqrt{ }$ & $\sqrt{ }$ & 6 \\
\hline 4 & Deployment & & $\sqrt{ }$ & & & $\sqrt{ }$ & & $\sqrt{ }$ & & $\sqrt{ }$ & & 4 \\
\hline 5 & SituationalAwareness & & $\sqrt{ }$ & & & $\sqrt{ }$ & & & & & & 2 \\
\hline 6 & ResponseGoal & & & $\sqrt{ }$ & & $\sqrt{ }$ & $\sqrt{ }$ & & & $\sqrt{ }$ & $\sqrt{ }$ & 5 \\
\hline 7 & Rescue & $\sqrt{ }$ & & & & $\sqrt{ }$ & & $\sqrt{ }$ & & & $\sqrt{ }$ & 4 \\
\hline 8 & Disaster & $\sqrt{ }$ & $\sqrt{ }$ & & $\sqrt{ }$ & & & & $\sqrt{ }$ & & & 4 \\
\hline 9 & SituationAnalysis & & $\sqrt{ }$ & & $\sqrt{ }$ & $\sqrt{ }$ & & & $\sqrt{1}$ & & & 4 \\
\hline 10 & Incident & & & & & & & & $\sqrt{ }$ & & & 1 \\
\hline 11 & Coordination & & $\sqrt{ }$ & & $\sqrt{ }$ & $\sqrt{ }$ & & $\sqrt{ }$ & & $\sqrt{ }$ & & 5 \\
\hline 12 & Command & & $\sqrt{ }$ & & & $\sqrt{1}$ & & & & & & 2 \\
\hline 13 & Communication & & $\sqrt{ }$ & & & $\sqrt{ }$ & & $\sqrt{ }$ & & $\sqrt{ }$ & & 4 \\
\hline 14 & StandardOperatingProcedure & & & & $\sqrt{ }$ & & & & & $\sqrt{ }$ & & 2 \\
\hline 15 & Victim & & & & & & & & & & $\sqrt{ }$ & $\mathbf{0}$ \\
\hline 16 & EmergencyManagementTeam & $\sqrt{ }$ & $\sqrt{ }$ & & $\sqrt{ }$ & $\sqrt{ }$ & & $\sqrt{ }$ & & $\sqrt{ }$ & $\sqrt{ }$ & 7 \\
\hline 17 & EmergencyOperationCentre & & $\sqrt{ }$ & & & & & $\sqrt{ }$ & & & & 2 \\
\hline 19 & Aid & & & & $\sqrt{ }$ & $\sqrt{ }$ & & $\sqrt{ }$ & & & $\sqrt{1}$ & 4 \\
\hline 20 & InformationManagement & & $\sqrt{ }$ & & $\sqrt{ }$ & $\sqrt{ }$ & & $\sqrt{ }$ & & $\sqrt{ }$ & & 5 \\
\hline 22 & RefugeeShelter & & & & $\sqrt{ }$ & & & & & & $\sqrt{ }$ & 2 \\
\hline 23 & MassCasualtyManagement & & & & & $\sqrt{ }$ & & & & $\sqrt{ }$ & & 2 \\
\hline 24 & FoodAid & & & & $\sqrt{ }$ & & & $\sqrt{ }$ & $\sqrt{ }$ & & $\sqrt{ }$ & 4 \\
\hline 25 & MedicalAid & & $\sqrt{ }$ & & $\sqrt{ }$ & $\sqrt{ }$ & & $\sqrt{ }$ & $\sqrt{1}$ & & $\sqrt{ }$ & 6 \\
\hline
\end{tabular}

The DoC categorisation of all DMM concepts (for all four DMM classes including the Preparedness and the Recovery) is shown in Table 6: 19 concepts in DMM1.1 are 
categorised as 'Very Strong', 23 are 'Strong', 25 are 'Moderate', 13 are 'Mild' and 4 concepts are 'Very Mild'. The four very mild concepts are Property, NaturalSite, BuildingCodes and Land-UsePlanning. Including them in DMM requires a reassessment. BuildingCodes and Land-UsePlanning are deleted as they are deemed as too specific to one kind of disaster (Bushfires). By revisiting DMM, it is found that the StructuralMitigation is in fact more generic to represent the BuildingCodes and Land-UsePlanning. As for the other two (Property and NaturalSite), they are opted to be kept as they are common across varying disasters.

Table 6. Degree of Confidence of DMM Concepts after FBS

\begin{tabular}{|c|c|}
\hline $\begin{array}{c}\text { DoC } \\
\text { Classification } \\
\end{array}$ & DMM Concepts \\
\hline $\begin{array}{c}100-70 \% \\
\text { (Very } \\
\text { Strong) }\end{array}$ & $\begin{array}{l}\text { MitigationPlan, MitigationOrganisation, MitigationTask, RiskReduction, } \\
\text { Resilience, HazardAssessment, RiskAnalysis, PreparednessPlan, } \\
\text { PreparednessOrganisation, EmergencyPublicInformation, ResponseOrganisation, } \\
\text { RecoveryPlan, Reconstruction, EmergencyManagementTeam, EmergencyPlan, } \\
\text { RecoveryOrganisation, RecoveryTask, DamageAssessment, } \\
\text { MentalHealthRecovery }\end{array}$ \\
\hline $\begin{array}{c}69-50 \% \\
\text { (Strong) }\end{array}$ & $\begin{array}{l}\text { MitigationGoal, DisasterRisk, StrategicPlanningOrganisation, PreparednessTask, } \\
\text { Warning, PreparednessGoal, Evacuation, BeforeDisaster, DisasterFactor, Training, } \\
\text { Media, PublicAwareness, Resource, Monitoring, ResponseTask, ResponseGoal, } \\
\text { Coordination, InformationManagement, MedicalAid (modify), RecoveryGoal, } \\
\text { After-Disaster, EconomicRestoration, FinancialAssistance }\end{array}$ \\
\hline $\begin{array}{c}49-30 \% \\
\text { (Moderate) }\end{array}$ & $\begin{array}{l}\text { NeedsPlanning, InformationUpdates, People, Lifeline, Vulnerability, Event, Effect, } \\
\text { SuppliesRegistry, DecisionMaking, Administration, Pre-Position, PublicEducation, } \\
\text { AidAgency, Deployment, Rescue, Disaster, SituationAnalysis, Communication, } \\
\text { Aid, FoodAid (modify), Demobilization, LongTermPlanning, TaskReview, } \\
\text { Exposure AidDistribution, }\end{array}$ \\
\hline $\begin{array}{c}29-11 \% \\
\text { (Mild) }\end{array}$ & $\begin{array}{l}\text { StructuralMitigation, Non-Structural Mitigation, Legislation, Insurance, Victim, } \\
\text { MutualAidAgreement, SituationAwareness, Command, MassCasualtyManagement } \\
\text { StandardOperatingProcedure, EmergencyOperationCentre, Incident, } \\
\text { RefugeeShelter (modify), }\end{array}$ \\
\hline $\begin{array}{c}10-0 \% \\
\text { (Very Mild) }\end{array}$ & Property $(\sqrt{ })$, NaturalSite $(\sqrt{ })$, BuildingCodes $(x)$, Land-UsePlanning (x) \\
\hline
\end{tabular}

The changes made to DMM1.1 here are affecting only the Mitigation-phase and Response-phase classes of concepts. Preparedness and Recovery-phase classes of concepts of DMM1.1 do not change here. New extension to the terminology is used to define three new concepts in the Response-phase class:

1) HumanitarianAid - Material or logistical assistance provided for humanitarian purposes, typically in response to an event or series of events which represents a critical threat to the health, safety, security or wellbeing of a community or other large groups of people, usually over a wide area.

2) DevelopmentAid - Aid to support the economic, environmental, social and political development of developing countries.

3) BilateralAid - Aid or funds that are given to one country from another. Since two concepts (BuildingCode and Land-UsePlanning) have been deleted in the second evaluation (Figure 6), the association relationships of 'isAGroupOf' owned by these concepts (in DMM1.1) are also deleted. The new version, DMM1.2, 
incorporates these changes as shown in Figures 6 (Mitigation-phase class) and 7 (Response-phase class).

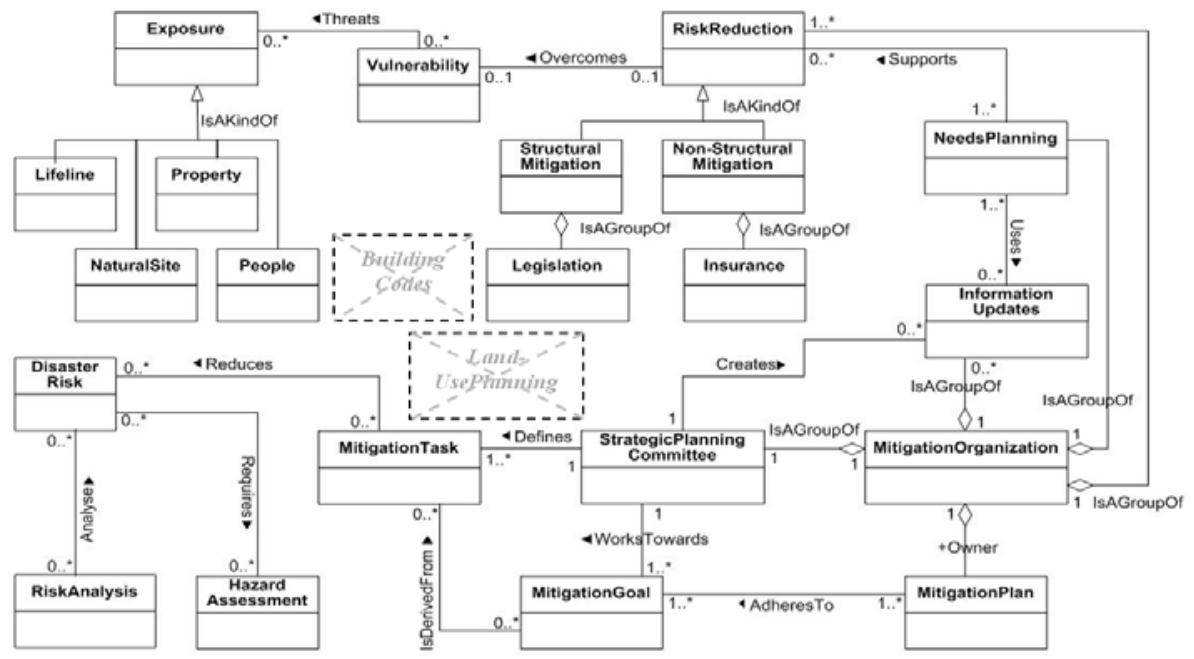

Fig. 6. The DMM1.2: A validated version of Mitigation-phase class of concepts

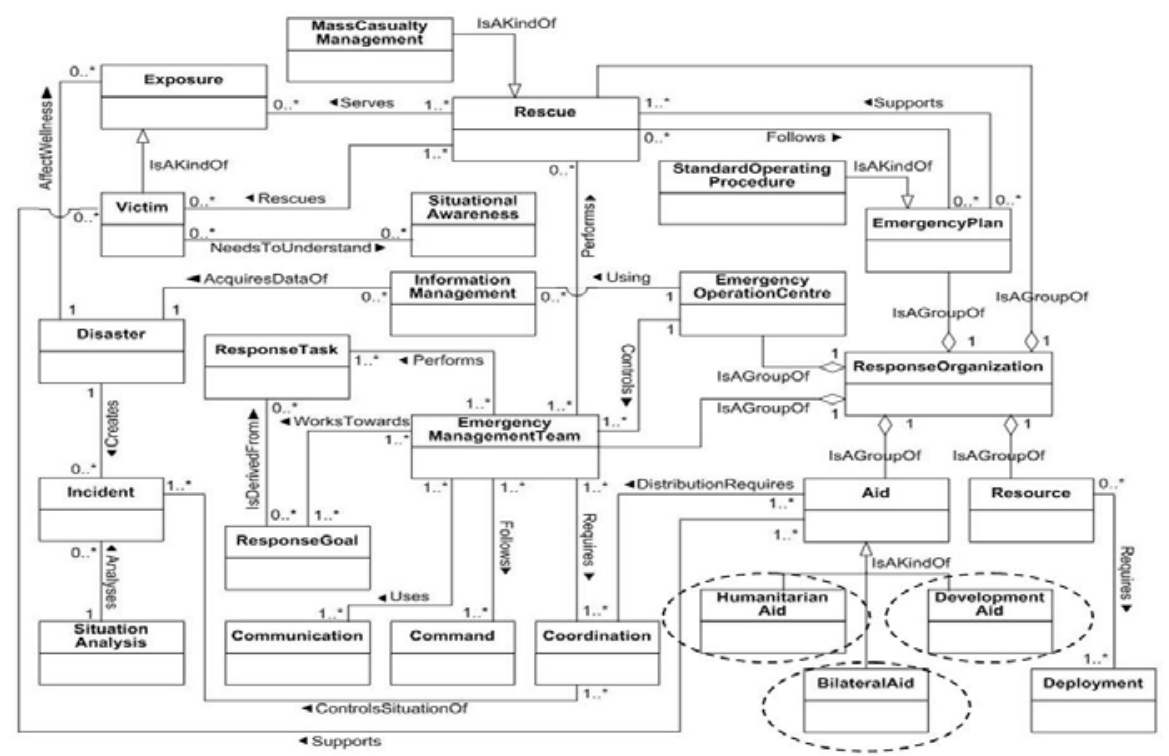

Fig. 7. The DMM1.2: A validated version of Response-phase class of concepts

\section{Conclusion}

In this paper, the evaluation of the Disaster Management Metamodel (DMM) is undertaken using the Frequency-based Selection (FBS) technique. To perform the FBS evaluation, a set of $10 \mathrm{DM}$ models is formed as a validation set (based on wider 
coverage to provide overlaps and to enable a frequency count of the individual DMM concepts). As a result from this evaluation, 3 concepts (HumanitarianAid, BilateralAid and DevelopmentAid) are added and 2 concepts are deleted (BuildingCode and Land-UsePlanning) from DMM. These changes are realised in DMM1.2. In addition, two concept relationships (aggregation - 'isAGroupOf') are also been deleted.

After performing the evaluation, the objective to evaluate the importance of the individual concepts used in each phase class of DMM is achieved. DMM is further improved. Its expressiveness and completeness of its concepts are enhanced. By deploying a proven evaluation method from the knowledge based community to metamodelling as used by software engineers, the paper makes an original contribution to both the $\mathrm{KB}$ and the $\mathrm{SE}$ communities. The use of metamodelling has hitherto being characterised by lack of systematic evaluation. By focussing on the evaluation and the metamodelling process on disaster management, this paper makes a significant contribution to this important domain unifying key concepts into a metamodel that can be used as knowledge sharing platform. Future works will develop a system prototype to demonstrate the applicability of the metamodel (DMM) in real world scenarios of disaster management.

\section{References}

1. Beydoun, G., Lopez-Lorca, A.A., Sanchez, F.G., Martinez-Bejar, R.: How do we measure and improve the quality of a hierarchical ontology? J. Syst. Softw. 84, 2363-2373 (2011)

2. Sprinkle, J.M.: Metamodel Driven Model Migration. vol. Doctor of Philosophy, pp. 176. Vanderbilt University, Tennessee, US (2003)

3. Gharehdaghli, A.: Design of a Generic Metamodel for Fieldwork Data Management. International Institute for Geo-Information, Science and Earth Observation vol. Master Theses. Netherlands, Enschede (2003)

4. Beydoun, G.: Formal concept analysis for an e-learning semantic web. Expert Syst. Appl. 36, 10952-10961 (2009)

5. Norris, D.M., Jon Mason, R.R., Lefrere, P., Collier, G.: A Revolution In Knowledge Sharing. EDUCAUSE review, vol. September October, (2003)

6. Smith, W., Dowell, J.: A case study of co-ordinative decision-making in disaster management. Ergonomics 43, 1153-1166 (2000)

7. Beerli, A.J., Falk, S., Diemers, D.: Knowledge Management and Networked Environments: Leveraging Intellectual Capital in Virtual Business Communities. AMACOM Books, New York (2003)

8. OMG: Unified Modelling Language Infrastructure Specification, Version 2.0, OMG document ptc/03-09-15.8,10,12, 13. (2004)

9. Trabelsi, C., Atitallah, R.B., Meftali, S., Dekeyser, J.-L., Jemai, A.: A Model-Driven Approach for Hybrid Power Estimation in Embedded Systems Design. EURASIP Journal on Embedded Systems 2011, 15 (2011)

10. W3C Incubator Group: 2008: Gary Berg-Cross, E.S.T., Rebecca Curzon, I., Chamindra de Silva, L.S.F., Paola Di Maio, U.o.S., Cutter Consortium , Renato Iannella, N., Mandana Sotoodeh, U.o.B.C., Olle Olsson, S., Guido Vetere, I.I.: http://www.w3.org/2005/Incubator/eiif/XGRFramework-20090806/\#ack

11. Garcia, P.B.: A Metamodel To Annotate Knowledge Based Engineering Codes As Enterprise Knowledge Resources. vol. PhD pp. 489. Cranfield University (2007)

12. Lagerstrom, R., Johnson, P., Hook, D.: Architecture analysis of enterprise systems modifiability - Models, analysis, and validation. Journal of Systems and Software 83, 1387-1403 (2010)

13. Vytautas Stuikys, R.D., Aleksandras Targamadze: A Model-Driven View To Meta-Program Development Process. Information Technology and Control 39, (2010) 
14. Falkenberg, E.D., Hesse, W., Lindgreen, P., Nilsson, B.E., Oei, J.L.H., Rolland, C., Stamper, R.K., Assche, F.J.M.V., Verrijn-Stuart, A.A., Voss, K.: A Framework of Information System Concepts, The FRISCO Report. University of Leiden (1998)

15. Gardner, T., Griffin, C., Koehler, J., Hauser, R.: A review of OMG MOF 2.0 Query / Views / Transformations submissions and recommendations towards the final standard. Workshop on Metamodeling for MDA, pp. 179-197 (2003)

16. Bieman, R.F.a.J.M.: Multi-View Software Evolution: A UML-based Framework for Evolving Object-Oriented Software. In: Proceedings International Conference on Software Maintenance (ICSM 2001). (2001)

17. Beydoun, G., Low, G., Henderson-Sellers, B., Mouraditis, H., Sanz, J.J.G., Pavon, J., GonzalesPerez, C.: FAML: A Generic Metamodel for MAS Development. IEEE Transactions on Software Engineering 35, 841-863 (2009)

18. Beydoun, G., Gonzalez-Perez, C., Henderson-Sellers, B., Low, G.C.: Developing and Evaluating a Generic Metamodel for MAS Work Products. In: A. Garcia et. al (ed.) Software Engineering for Multi-Agent Systems IV: Research Issues and Practical Applications, vol. LNCS 3914, pp. 126-142. Springer-Verlag, Berlin (2006)

19. Othman, S.H., Beydoun, G.: Metamodelling Approach To Support Disaster Management Knowledge Sharing. Australasian Conference on Information Systems (ACIS'2010) Proceeding, Paper 97, Brisbane, Australia (2010)

20. Othman, S.H., Beydoun, G.: A Disaster Management Metamodel (DMM) Validated. In: Kang, B.-H., Richards, D. (eds.) 11th International Workshop on Knowledge Management and Acquisition for Smart Systems and Services (PKAW'2010), vol. LNAI 6232, pp. 111-125. Springer-Verlag, Berlin, Daegu, Korea (2010)

21. Kok, D.d.: Feature Selection for Fluency Ranking. In: Proceedings of the SixthInternational Natural LanguageGeneration Conference (INLG 2010). (2010)

22. Zhang, Z., Ye, N.: Locality preserving multimodal discriminative learning for supervised feature selection. Knowledge and Information Systems 27, 473-490 (2011)

23. Davis, P.K., Bigelow, J.H.: Motivated Metamodels. In: Proceedings of the 2002 Performance Metrics for Intelligent Systems Workshop (PerMIS' 02). (2002)

24. Ahmed, I.: Disaster Risk Management Framework. In: International Training Workshop on Disaster Risk \& Environmental Management. (2008)

25. Cecilia Rosenberg (FEMA): Chapter 3: Policies for Guiding Planning for Post-Disaster Recovery and Reconstruction. Appear in PAS Report No. 483/484 by American Planning Association, (2005)

26. Garatwa, W., Bollin, C.: Disaster Risk Management : Working Concept. (2002)

27. Ulrich Boes, U.L.: Disaster Information, Innovative Disaster Information Services. (2008)

28. Buford, J.F., Jakobson, G., Lewis, L.: Multi-Agent Situation Management for Supporting LargeScale Disaster Relief Operations. International Journal of Intelligent Control and Systems 11, 284-295 (2006)

29. Mendis, D.S.K., Karunananda, A.S., Samaratunga, U., Ratnayake, U.: An Approach to the Development of Commonsense Knowledge Modeling Systems for Disaster Management. Artificial Intelligence Review 28, 179-196 (2007)

30. A. Coburn, Spencer, R.: Earthquake Protection. Wiley Inc, Chichester-New York (1992)

31. Shaluf, I.M.: Technological Disaster Stages and Management. Journal of Disaster Prevention and Management 17, 114-126(2008)

32. Jennings-Sanders, A.: Teaching disaster nursing by utilizing the Jennings Disaster Nursing Management Model. Nurse Education in Practice 4, 69-76 (2004)

33. Khan, H., Vasilescu, L.G., Khan, A.: Disaster Management Cycle - A Theoretical Approach. Journal of Management and Marketing 6, 43-50 (2008)

34. Kelly, S., Pohjonen, R.: Worst Practices for Domain-Specific Modeling. IEEE Software 26, 2229 (2009) 